\title{
ARTIGO \\ Estudo da Interferência dos Déficits ORIGINAL Motor e Sensitivo na Função Manual de Pacientes Hemiplégicos Submetidos à Estimulação Elétrica Funcional (FES)*
}

\author{
Margarida Harumi Miyazaki** \\ Maria Inês Lourenção*** \\ José Brenha Ribeiro Sobrinho**** \\ Claudete Lourenço ${ }^{* * * *}$ \\ Linamara Rizzo Battistella*****
}

\section{RESUMO}

O presente estudo procura identificar os aspectos fisiopatológicos que condicionam um mau prognóstico na aplicação da Estimulação Elétrica Funcional $^{1}$ (FES), sendo pareados os bons e maus resultados com os déficits motor e sensitivo. Foram avaliados 17 pacientes hemiplégicos, com praxia e gnosia preservados, submetidos às técnicas convencionais da Terapia Ocupacional e Estimulação Elétrica Funcional. Os eletrodos foram ajustados, de modo a obter dorsiflexão de punho e dedos e abdução de polegar, quando possível. Foram realizadas avaliações das sensibilidades superficial (tactil e dolorosa) e profunda (reconhecimento de diferentes posições do membro acometido), das preensões tipo cilindro, esfera, gancho e pinças lateral, polpa a polpa e 03 pontos, antes e logo após 06 meses de FES. Resultados mostraram que 13 pacientes obtiveram melhora da movimentação ativa manual. Os pacientes que melhoraram tinham sensibilidade superficial e profunda normal ou alterada, mas apresentavam algum tipo de movimentação manual espontânea. Os pacientes, que não melhoraram, tinham alterações das sensibilidades superficial e/ou profunda, mas não tinham qualquer movimentação manual ativa. Conclui-se que a associação da Estimulação Elétrica Funcional às técnicas convencionais de Terapia Ocupacional é um meio eficaz de melhorar a função manual de pacientes hemiplégicos com movimentação voluntária parcialmente preservada.

\section{UNITERMOS}

FES. Déficit motor. Déficit Sensitivo. Função Manual. Hemiplegia.

\footnotetext{
* Trabalho realizado na Divisão de Medicina de Reabilitação - HCFMUSP

** Médico Supervisor de Equipe - Divisão de Medicina de Reabilitação HCFMUSP.

** Terapeuta Ocupacional Chefe do Serviço de Terapia Ocupacional - Divisão de Medicina de Reabilitação - HCFMUSP.

**** Médica Fisiatra - Diretora do Serviço Médico - Divisão de Medicina de Reabilitação - HCFMUSP.

**** Médica Fisiatra, Diretora do Serviço Médico da Divisão de Medicina de Reabilitação - HUFMUSP, e Vice-Presidente da Soc. Bras. de Med. Física e Reabilitação

***** Professora Doutora, Diretora da Divisão de Medicina de Reabilitação HCFMUSP.
} 


\section{SUMMARY}

This study was performed to identify the physiophatologic aspects that lead to a bad prognosis in Functional Electric Stimulation (FES) in hemiplegic patients. There were compared good and bad results concerning to the motor and sensory deficits. 17 hemiplegic patients non apraxic and non agnosic were evaluated. They were submitted to conventional techniques of ocupational therapy and FES, for least 6 months in wrist and fingers dorsiflexion and if it was possible, in the thumb abduction, too. It was mode sensory evaluation (superficial and deep), before the treatment and after 6 months. The results were that 13 patients improved that active manual movements. Those who improved had normal or altered sensibility, both superficial as deep, but they had some spintaneous manual movements. Those who didn't improve had altered sensibility, either superficial or deep, but they didn't have any spontaneous manual movements. It was conclued that the association of FES to the conventional technics of ocupational therapy are effective in improving manual function in hemiplegic patients with voluntary movements parcially preserved.

\section{KEY WORDS}

Functional Electrion Stimulation. Sensory impairment. Motor impairment. Manual function. Hemiplegia.

\section{Introdução}

No paciente hemiplégico, raramente o membro superior é preservado, o que contribui para a incapacidade funcional ${ }^{2}$, levando A dificuldades na realização das atividades de vida diária, prática e profissional. Assim, procuramos ampliar a utilização de recursos que possam melhorar o processo de reabilitação. Dentre eles, temos o FES, cuja aplicação procura melhorar a restauração da função perdida ${ }^{3}$. $\mathrm{O}$ nosso objetivo

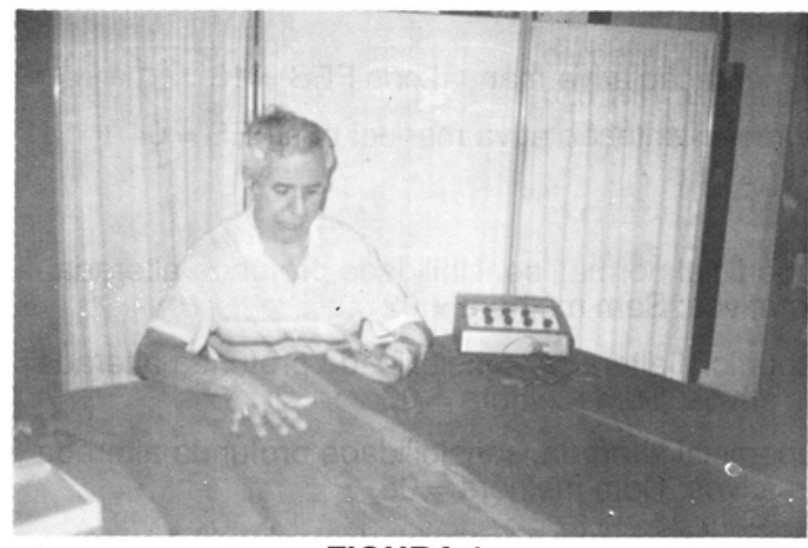

FIGURA 1

Aplicação da FES em membro superior acometido é estudar a influência das sensibilidadeS superficial e profunda e dos movimentos ativos na reabilitação da função manual do membro acometido do paciente hemiplégico quando as técnicas convencionais de terapia ocupacional são associadas à estimulação elétrica funcional.

TABELA 1 - CASUÍSTICA

\begin{tabular}{|c|c|c|}
\hline 17 Pacientes & & \\
\hline \multirow[t]{2}{*}{ Sexo } & Masculino & 12 \\
\hline & Feminino & 5 \\
\hline \multirow[t]{3}{*}{ Idade } & Mínimo & $15 \mathrm{a}$ \\
\hline & Máximo & $69 a$ \\
\hline & Média & $48,7 \mathrm{a}$ \\
\hline \multirow[t]{3}{*}{ Tempo de AVC } & Mínimo & $6 \mathrm{~m}$ \\
\hline & Máximo & 13 a $9 \mathrm{~m}$ \\
\hline & Médio & $28,35 \mathrm{~m}$ \\
\hline \multirow[t]{2}{*}{ Incapacidade } & D - 8 & \\
\hline & E - 9 & \\
\hline \multirow[t]{2}{*}{ Dominância } & D - 17 & \\
\hline & $\mathrm{E}-0$ & \\
\hline \multirow[t]{2}{*}{ Gnosia } & Alterado & 0 \\
\hline & Normal & 17 \\
\hline \multirow[t]{2}{*}{ Praxia } & Alterada & 0 \\
\hline & Normal & 17 \\
\hline \multirow[t]{4}{*}{ Sensibilidade } & Sup. Alterada & 8 \\
\hline & Normal & 9 \\
\hline & Prof. Alterada & 10 \\
\hline & Normal & 7 \\
\hline \multirow{2}{*}{$\begin{array}{c}\text { Movimentação } \\
\text { Ativa Manual }\end{array}$} & Presente & 13 \\
\hline & Ausente & 04 \\
\hline
\end{tabular}

\section{Material e Método}

Oestudo incluiu 17 pacientes, sendo 12 de sexo masculino e 5 do feminino, com idade média de 48,7 anos (15-69 anos). O tempo de Acidente Vascular Cerebral (AVC) variou de 6 a 28,35 m (média = 28,35 meses). Oito tinham acometimento do dimídio direito e nove do esquerdo. Todos eram dextros e possuíam gnosia e praxia preservadas. Quanto à sensibilidade, 8 indivíduos tinham alteração da superficial e 10 da sensibilidade profunda. Apenas 13 pacientes apresentavam movimentação manual ativa presente (tabela 1). 
Foram realizadas técnicas convencionais de Terapia Ocupacional e Estimulação Elétrica Funcional. O equipamento utilizado foi o eletroestimulador Quadrikron KC-170 (Fig. 1). Utilizamos um canal de estimulação. Os eletrodos foram colocados na face dorsal e/ou lateral do antebraço acometido e os parâmetros foram regulados, de modo a obtermos o melhor padrão de dorsiflexão de punho e dedos e oponência ou abdução de polegar. A duração do estímulo foi de 30 minutos, concomitante ou não à realização das atividades de Terapia Ocupacional, numa frequência de 2-3 vezes por semana. Foram feitas avaliações das sensibilidades superficial (táctil e dolorosa) e profunda (reconhecimento de diferentes posições do membro acometido), das preensões grosseiras tipo cilindro, esfera, gancho, e pinças lateral, polpa a polpa, ponta a ponta e três pontos, antes e após seis meses de FES. Quanto à presença de preensões e pinças, ambas foram graduadas em ausente, esboço, parcial e normal.

\section{Resultado}

Os resultados mostraram que 13 pacientes obtiveram melhora da movimentação ativa manual após FES, sendo que todos tinham alguma mobilidade, funcional ou não, prévia ao tratamento. Essa melhora foi independente a distúrbio de sensibilidade presente (Fig. 2).

\section{Discussão}

O presente trabalho procurou identificar os aspectos fisiopatológicos que condicionam um bom diagnóstico na aplicação de Estimulação Elétrica Funcional, sendo pareados os bons e maus resultados com os déficits motores e sensitivos. Como pode ser visto, os pacientes beneficiados com o tratamento foram os que apresentaram movimentação ativa preservada, ainda que de maneira parcial. Os déficits sensoriais podem eventualmente serem compensados pela reorganização do "campo aferente", com aumento da participação de aferência visual na retroalimentação reguladora dos movimentos, não sendo, como foi observado, fator limitante de melhora.

\section{Conclusão}

A estimulação elétrica Funcional é um meio eficaz na melhora da função manual de pacientes hemiplégicos com movimentação voluntária parcialmente preservada.

\section{Referências Bibliográficas}

1. GRACANIN F. - Aplicação de Corrente de Baixa Freqüência em Medicina Física e Reabilitação com Ênfase Especial em Estímulo Elétrico Funcional. Medicina de Reabilitação. 20/21: 18-23, 1988.

2. NAKAYAMA H., Jorgensen H. S., RAAS CHOU H.O., OLSEN, T.J. - Recovery of Upper Extremity Function in Stroke Patients: the Copenhagen Stroke Study. Arch Phys Med. Reabil. 75: 394-98, 1994.

3. KRALJA, ALIMOVIE R., STANIC, U. - Enhancement of Hemiplegic Patient Rehabilitation by Means of Functional Electrical Stimulation. Prosthetics and Orthotics International, 17: 107-114, 1993.

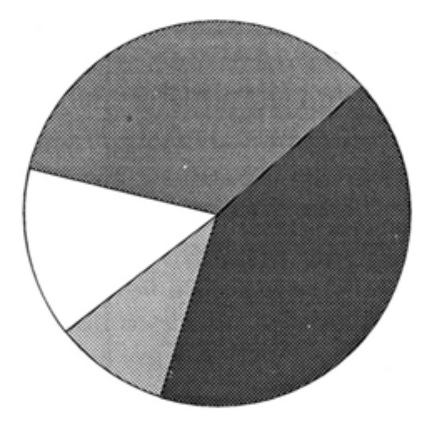

Melhora da movimentação ativa manual pós FES $=13$

Sem melhora da movimentação ativa manual pós FES $=04$

Sensibilidade superficial normal, sensibilidade profunda alterada sem movimentos ativos: Sem melhora $=02$

Sensibilidade superficial alterada, sensibilidade profunda alterada sem movimentos ativos: Sem melhora $=02$

Sensibilidade superficial alterada, sensibilidade profunda alterada com movimentos ativos: Com melhora $=06$

Sensibilidade superficial normal, sensibilidade profunda normal com movimentos ativos: Com melhora $=07$ 